\title{
Erratum to: Peak velocities estimation for a direct five- step design procedure of inter-storey viscous dampers
}

\section{Michele Palermo ${ }^{1}$ - Stefano Silvestri ${ }^{1}$. Luca Landi ${ }^{1}$. \\ Giada Gasparini ${ }^{1}$ - Tomaso Trombetti ${ }^{1}$}

Published online: 14 December 2015

(C) Springer Science+Business Media Dordrecht 2015

Unfortunately, there are mistakes in Eqs. (20-23). The corrected equations are given here:

$$
\begin{aligned}
c_{N L, A}= & c_{L} \cdot\left(0.8 \cdot v_{\max , \mathrm{A}} \cdot \cos \theta\right)^{1-\bar{\alpha}} \\
= & \bar{\xi} \cdot \omega_{1} \cdot m_{\text {tot }} \cdot\left(\frac{N+1}{n}\right) \cdot \frac{1}{\cos ^{2} \theta} \cdot\left(M \cdot 0.8 \cdot \frac{S_{a}}{\omega_{1}} \cdot \frac{12 N}{\left(2+5 N+5 N^{2}\right)} \cdot \cos \theta\right)^{1-\bar{\alpha}} \\
c_{N L, B} & =c_{L} \cdot\left(0.8 \cdot v_{\max , \mathrm{B}} \cdot \cos \theta\right)^{1-\bar{\alpha}} \\
& =\bar{\xi} \cdot \omega_{1} \cdot m_{\text {tot }} \cdot\left(\frac{N+1}{n}\right) \cdot \frac{1}{\cos ^{2} \theta} \cdot\left(M \cdot 0.8 \cdot \frac{S_{a}}{\omega_{1}} \cdot \frac{2}{N+1} \cdot \cos \theta\right)^{1-\bar{\alpha}}
\end{aligned}
$$

where the factor $\cos \theta$ is applied to $v_{\max , \mathrm{A}}$ and $v_{\max , \mathrm{B}}$ to obtain the velocity along the inclined direction of the damper.

$$
\begin{gathered}
F_{N L, A}=c_{N L, A} \cdot v_{\max , \mathrm{A}}^{\bar{\alpha}}=0.8^{1-\bar{\alpha}} \cdot \bar{\xi} \cdot m_{t o t} \cdot \frac{1}{n \cdot \cos \theta} \cdot M \cdot S_{a}\left(T_{1}, \bar{\xi}\right) \cdot \frac{12 N(N+1)}{\left(2+5 N+5 N^{2}\right)} \\
F_{N L, B}=c_{N L, B} \cdot v_{\max , \mathrm{B}}^{\bar{\alpha}}=2 \cdot 0.8^{1-\bar{\alpha}} \cdot \bar{\xi} \cdot m_{t o t} \cdot \frac{1}{n \cdot \cos \theta} \cdot M \cdot S_{a}\left(T_{1}, \bar{\xi}\right)
\end{gathered}
$$

The online version of the original article can be found under doi:10.1007/s10518-015-9829-8.

Michele Palermo

michele.palermo7@unibo.it

1 Department DICAM, University of Bologna, Viale Risorgimento 2, 40136 Bologna, Italy 